\title{
Preparation of porous scaffolds by using freeze-extraction and freeze-gelation methods
}

\author{
Ming-Hua $\mathrm{Ho}^{\mathrm{a}}$, Pei-Yun Kuo ${ }^{\mathrm{a}}$, Hsyue-Jen Hsieh ${ }^{\mathrm{a}}$, Tzu-Yang Hsien ${ }^{\mathrm{b}}$, Lein-Tuan $\mathrm{Hou}^{\mathrm{c}}$, \\ Juin-Yih Lai ${ }^{\mathrm{d}}$, Da-Ming Wang ${ }^{\mathrm{a}, *}$ \\ a Department of Chemical Engineering, National Taiwan University, Taipei 106, Taiwan \\ ${ }^{\mathrm{b}}$ Environmental Protection Administration, Government of ROC, Taipei, Taiwan \\ ${ }^{\mathrm{c}}$ Department of Periodontology, School of Dentistry, National Taiwan University, Taipei, Taiwan \\ ${ }^{\mathrm{d}} R \& D$ Center for Membrane Technology, Department of Chemical Engineering, Chung Yuan University, Chung Li, Taiwan
}

Received 27 November 2002; accepted 15 June 2003

\begin{abstract}
Freeze-fixation and freeze-gelation methods are presented in this paper which can be used to prepare highly porous scaffolds without using the time and energy consuming freeze-drying process. The porous structure was generated during the freeze of a polymer solution, following which either the solvent was extracted by a non-solvent or the polymer was gelled under the freezing condition; thus, the porous structure would not be destructed during the subsequent drying stage. Compared with the freeze-drying method, the presented methods are time and energy-saving, with less residual solvent, and easier to be scaled up. Besides, the problem of formation of surface skin can be resolved and the limitation of using solvent with low boiling point can be lifted by the presented methods. With the freeze-extraction and freeze-gelation methods, porous PLLA, PLGA, chitosan and alginate scaffolds were successfully fabricated. In addition to the presentation of the morphologies of the fabricated scaffolds, preliminary data of cell culture on them are as well included in the present work.
\end{abstract}

(C) 2003 Elsevier Ltd. All rights reserved.

Keywords: Scaffold; Freeze-extraction; Freeze-gelation; Freeze-drying; Tissue engineering

\section{Introduction}

Tissue engineering, a technique to create new tissue from cultured cells, has now been considered as a potential alternative to organ or tissue transplantation [1]. Biodegradable scaffolds, serving as transplant vehicles for cultured cells and templates to guide tissue regeneration, play an important role in transforming the cultured cells to a new tissue [1-3]. To allow a high density of seeded cells and to promote neovascularization when being implanted in vivo, the scaffolds should have high porosity, large surface area, suitable pore size, and highly interconnected pore structure, in addition to biocompatibility and biodegradability [4-7]. Therefore, a lot of researches have concentrated on developing

*Corresponding author. Tel.: +886-2-23660433; fax: +886-223623040.

E-mail address: daming@ccms.ntu.edu.tw (D.-M. Wang). methods for preparing scaffolds with suitable porous structure for tissue engineering.

Several preparation methods have been reported for porous scaffolds, including porogen leaching [8-10], saturation and release of $\mathrm{CO}_{2}$ [11,12], 3D printing [13], and phase separation techniques [10,14-18]. A widely used method is the thermally induced phase separation (TIPS) $[10,14,15]$, in which the solution temperature is lowered to introduce phase separation of the homogeneous biodegradable polymer solution. The phase separation mechanism could be liquid-liquid demixing [16], which generates polymer-poor and polymer-rich liquid phases. The subsequent growth and coalescence of the polymer-poor phase would develop to form the pores in scaffolds. On the other hand, when the temperature is low enough to allow the freeze of the solution, the phase separation mechanism would be solid-liquid demixing, which forms frozen solvent and concentrated polymer phases. After the removal of the 
frozen solvent, the remained space would become pores. By adjusting the polymer concentration, using different solvent, or varying the cooling rate, phase separation could occur via different mechanisms, resulting in scaffolds with various morphologies.

The porous structure is in fact generated in the stage of phase separation. After the removal of the liquid or frozen solvent contained in the demixed solution, the space originally occupied by the solvent would become pores in the prepared scaffolds. Obviously, in the stage of solvent removal, the porous structure contained in the solution needs to be carefully retained. Therefore, freeze-drying is usually used for solvent removal to retain the porous structure. Without freeze-drying, a rise in temperature during the drying stage could result in remixing of the phase-separated solution or remelting of the frozen solution, leading to a destruction of the porous structure. Although freeze-drying can prevent the disintegration of porous structure, it is so time and energy consuming that the whole scaffold fabrication process becomes inefficient and economically uncompetitive. Another problem encountered in the application of freeze-drying to preparation of scaffolds is the occurrence of surface skin. During the freeze-drying stage, if the temperature is not controlled low enough, the polymer matrix would not be rigid enough to resist the interfacial tension caused by evaporation of solvent. Thus, the porous structure collapses and dense skin layers occur in the prepared scaffolds. To improve the efficiency of the fabrication process and to prevent the formation of surface skin, a method is presented in this paper which fixes the porous structure under freezing condition so that in the subsequent drying stage the freeze-drying process is not needed.

The fabrication procedures for four biodegradable polymers are discussed in the present work, containing poly (L-lactic acid) (PLLA), poly (lactic-co-glycolic acid) (PLGA), chitosan, and alginate. For PLLA and PLGA, after the polymer solution was frozen, the frozen solution was immersed in a non-solvent bath to allow the exchange of solvent and non-solvent at a temperature lower than the freezing point of the polymer solution. Such a freeze-extraction technique can extract the solvent out of the polymer matrix before the drying stage. After that, the polymer matrix was surrounded with non-solvent, redissolution of polymer would not occur in the drying stage and freeze-drying was therefore not needed. On the other hand, for chitosan and alginate, the frozen polymer solution was immersed in a gelation environment at a temperature lower than the freezing point of the polymer solution. With this freezegelation technique, since the polymer matrix had already gelled before the drying stage, the porous structure could also be retained without freeze-drying.

In the following, the procedures of freeze-extraction and freeze-gelation will be discussed. The prepared scaffolds will be compared with those fabricated by using the freeze-drying method. In addition, some preliminary data of cell culture on the prepared scaffolds will also be given.

\section{Materials and methods}

\subsection{Materials}

PLGA 50/50 (poly (D, L-lactic-co-glycolic acid) 50:50), chitosan and alginate were purchased from Sigma, PLLA (poly (L-latide)) from Fluka. All the solvents used were of analytical quality. Distilled and deionized water was used throughout this study. For cell culture, $\alpha$-minimum essential medium ( $\alpha$ MEM) was purchased from Sigma, and fetal bovine serum (FBS) and penicillin-streptomycin-amphotercin were purchased from Gibco.

\subsection{Preparation of PLLA and PLGA scaffolds}

PLLA was dissolved in dioxane and PLGA was dissolved in dioxane or DMSO (dimethyl sulfoxide) to form a $3 \mathrm{wt} \%$ polymer solution. The polymer solution was placed in a glass petridish and frozen at $-20^{\circ} \mathrm{C}$. The solvent contained in the frozen solution was then removed either by freeze-drying or by freeze-extraction. The procedures of freeze-extraction are described below. The frozen polymer solution was immersed in an ethanol aqueous solution that was pre-cooled to $-20^{\circ} \mathrm{C}$. It should be noted that the ethanol concentration was $80 \mathrm{wt} \%$ preparation of PLLA scaffolds, and $30 \mathrm{wt} \%$ for PLGA. Due to the miscibility between the solvent (dioxane or DMSO) and the ethanol aqueous solution, the solvent was extracted out and replaced with ethanol aqueous solution, a non-solvent for PLLA and PLGA. After extraction, drying at room temperature was performed to remove the ethanol aqueous solution contained in the polymer matrix. PLLA and PLGA scaffolds could then be obtained after the drying stage.

\subsection{Preparation of chitosan and alginate scaffolds}

Chitosan was dissolved in acetic-acid aqueous solution $(1 \mathrm{M})$ to form a $2 \mathrm{wt} \%$ polymer solution. The polymer solution was placed in a glass petridish and frozen at $-20^{\circ} \mathrm{C}$. The frozen chitosan solution was immersed in a $\mathrm{NaOH} /$ ethanol aqueous solution to adjust its $\mathrm{pH}$ to allow for the gelation of chitosan. The $\mathrm{NaOH} /$ ethanol aqueous solution was precooled to $-20^{\circ} \mathrm{C}$ so that the gelation occurred below the freezing point of the chitosan solution; hence, the process is called "freeze-gelation". For comparison, freeze-drying 
was also conducted to remove the aqueous solution contained in the frozen chitosan solution.

The procedures to prepare alginate scaffolds are similar to those for chitosan scaffolds. Alginate was dissolved in deionized water to form a $2 \mathrm{wt} \%$ solution, which was then frozen at $-20^{\circ} \mathrm{C}$. The frozen solution was immersed in aqueous ethanol solution of $\mathrm{CaCl}_{2}$ at $-20^{\circ} \mathrm{C}$ to induce gelation of alginate. Drying at room temperature was performed after gelation to obtain alginate scaffolds.

\subsection{Freeze-drying}

As mentioned in Sections 2.2 and 2.3, freeze-drying was also performed in the present work for comparison with freeze-extraction and freeze-gelation. It should be noted that, when the commercial freeze-dryer was used, the prepared scaffolds possessed surface skin. To prevent the formation of surface skin, the apparatus depicted in Fig. 1 was used to carry out the freeze-drying process. During operation, liquid nitrogen was constantly added to the liquid nitrogen bath to prevent the elevation of temperature.

\subsection{Determination of the porosity of scaffolds}

Both the mass $(M)$ and the volume $(V)$ of the prepared scaffolds were measured. Dividing $M$ by the density of polymer $\left(\rho_{\mathrm{p}}\right)$ gave the volume of polymer $\left(V_{\mathrm{p}}\right)$ contained in the scaffolds. The polymer density can be obtained from the supplier: PLLA $1.25\left(\mathrm{~g} / \mathrm{cm}^{3}\right)$, PLGA $1.37\left(\mathrm{~g} / \mathrm{cm}^{3}\right)$, chitosan $0.50\left(\mathrm{~g} / \mathrm{cm}^{3}\right)$, alginate $0.90(\mathrm{~g} /$ $\mathrm{cm}^{3}$ ). With the above information, the porosity of scaffolds can be calculated via the following equation:

Porosity $=\frac{V-V_{\mathrm{p}}}{V} 100 \%$.

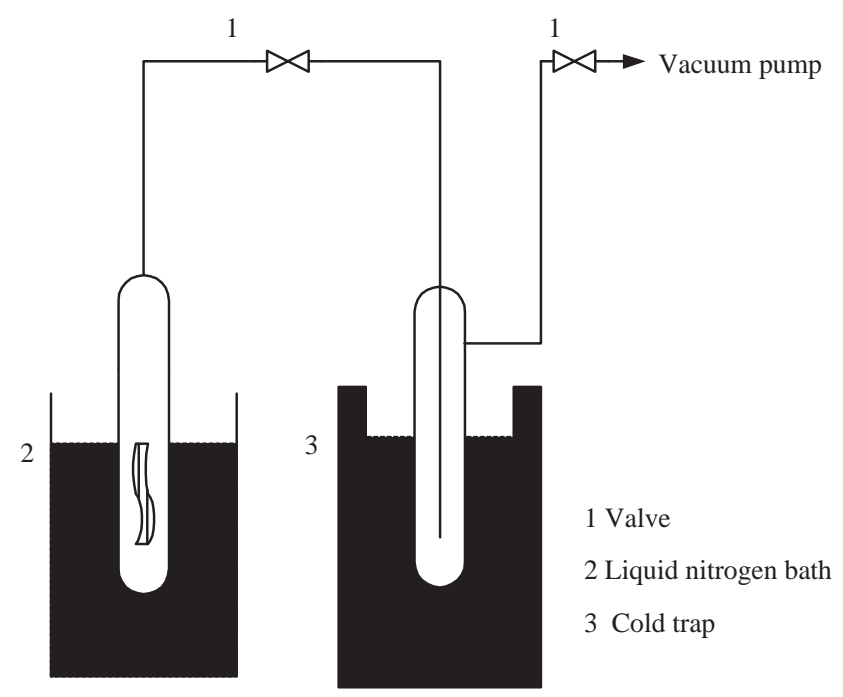

Fig. 1. Schematic diagram of a freeze-drying apparatus.

\subsection{Pore size distribution}

Pore size distribution of porous scaffolds was examined by using Mercury porosimetry (60 K-1-A, PMI).

\subsection{SEM analysis}

For analysis with SEM, specimens were cut from the scaffolds and fractured in liquid nitrogen. A gold layer was then coated on the specimen surface. Morphologies of specimens were examined by using JEOL (JSM-6300) scanning electron microscope. To prepare samples of cell-containing scaffolds for SEM analysis, fixation and dehydration of cells should be carried out. The scaffolds with cells were fixed with a $1 \mathrm{wt} \%$ glutaraldehyde solution and dehydrated in a series of ethanol aqueous solutions with successively increasing ethanol concentration from 30 to $99.99 \mathrm{wt} \%$. Critical point drying with supercritical $\mathrm{CO}_{2}$ was then performed to prevent the deformation of cells.

\subsection{Cell culture}

Cells used for culture were ROS 17/2.8 osteoblast-like cells, originally isolated from a rat osteosarcoma. The cells were cultured in $\alpha$ MEM, supplemented with $10 \%$ FBS and $100 \mathrm{U} / \mathrm{ml}$ penicillin-streptomycin-amphotercin, at $37^{\circ} \mathrm{C}$ in $5 \%$ of $\mathrm{CO}_{2}$. Scaffolds were placed and immobilized in culture dishes. ROS cells suspended in culture medium $\left(5 \times 10^{4}\right.$ cells $\left./ \mathrm{ml}\right)$ were then added in the dishes to allow the ingrowth of cells to the scaffolds. The culture medium was changed every 2 days. After incubation for various periods, cells attached on the scaffolds were harvested for analysis.

\subsection{Determination of cell density in scaffolds}

The scaffolds were washed with PBS for 3 times to remove the non-attached ROS cells and were then immersed into $0.5 \times$ trypsin solution and incubated at $37^{\circ} \mathrm{C}$ for $10 \mathrm{~min}$ to detach the cells for analysis. The number of cells was determined with a hemacytometer.

\section{Results and discussion}

\subsection{Scaffolds prepared by freeze-extraction and freeze- gelation methods}

From the micrographs shown in Figs. 2-5, it can be seen clearly that porous PLLA and PLGA scaffolds can be successfully fabricated by using the freeze-extraction method and chitosan and alginate scaffolds can be prepared by the freeze-gelation method. The PLLA and 


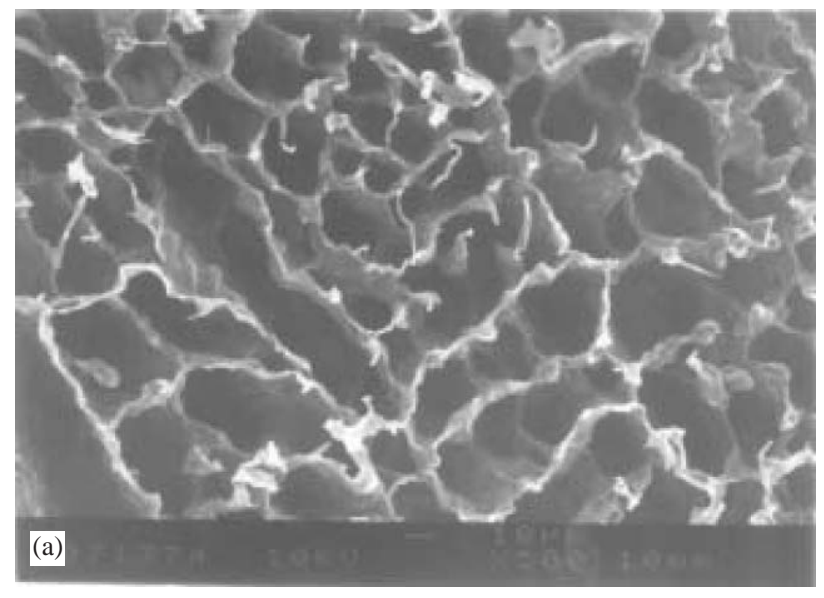

$-10 \mu \mathrm{m}$

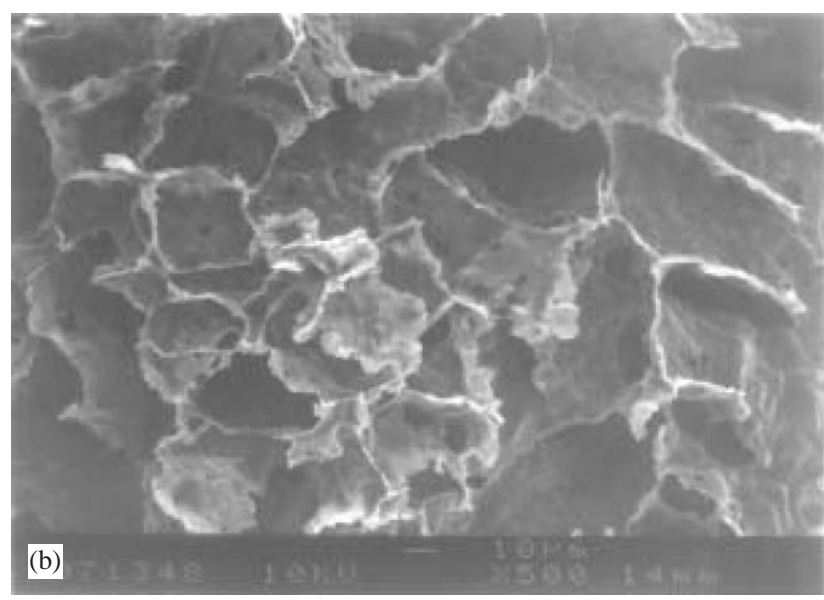

$-10 \mu \mathrm{m}$

Fig. 2. Morphology of the PLLA scaffold prepared by the freezeextraction method (SEM). Solvent: dioxane. (a) Surface and (b) crosssection.

PLGA scaffolds shown in Figs. 2 and 3 were prepared by, respectively, freezing the PLLA/dioxane and PLGA/ dioxane solutions at $-20^{\circ} \mathrm{C}$. The frozen solutions were immersed in ethanol aqueous solution at $-20^{\circ} \mathrm{C}$. The ethanol aqueous solution was then removed by drying at room temperature, leading to the formation of porous scaffolds. The chitosan scaffold in Fig. 4 was prepared by freezing the chitosan/acetic acid/water solution at $-20^{\circ} \mathrm{C}$, and the frozen solution was immersed in $\mathrm{NaOH} /$ ethanol/water solution at the same temperature to adjust the $\mathrm{pH}$ to allow for the gelation of chitosan. The liquid contained in the gel was then removed by drying at room temperature. The procedures for preparing alginate scaffolds are similar to those for chitosan scaffolds except that the polymer solvent was pure water and the frozen solution was immersed in a solution containing calcium ion to bring about the gelation of alginate.

It can be seen from Figs. 2-5 that the pores in the scaffolds are interconnected and range from 60 to
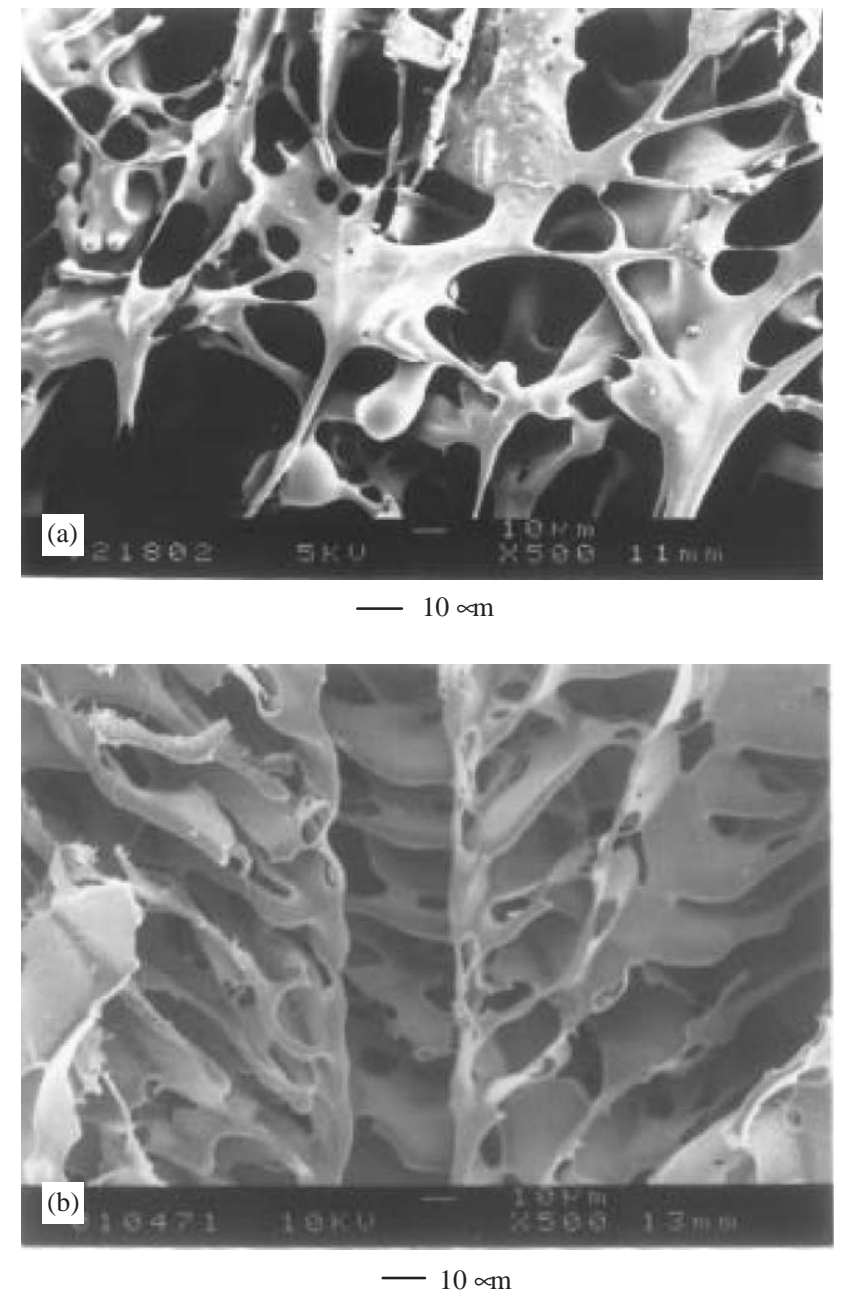

Fig. 3. Morphology of the PLGA scaffold prepared by the freezeextraction method (SEM). Solvent: dioxane. (a) Surface and (b) crosssection.

$150 \mu \mathrm{m}$. To demonstrate that the pore-size information obtained from SEM is reliable, mercury porosimetry was used to determine the pore size distribution. The results are depicted in Fig. 6, indicating that most pores are in the range of $60-150 \mu \mathrm{m}$, similar to the estimations rom SEM. Obviously, the scaffolds prepared by freezeextraction and freeze-gelation possess suitable pore size for application to tissue engineering [1-7], and the interconnected pore structure should be able to provide enough space for possible neovascularization when being implanted. Besides, the porosity of the scaffolds was also measured and the results are shown in Fig. 7. The data indicate that the porosity of the prepared scaffolds is high enough $(>80 \%)$ for usage in tissue engineering.

For comparison we also performed experiments of which the solvent was removed by freeze-drying. Scaffolds were prepared by freezing the polymer solutions at $-20^{\circ} \mathrm{C}$. The frozen solutions were then freeze-dried to prevent the destruction of porous 

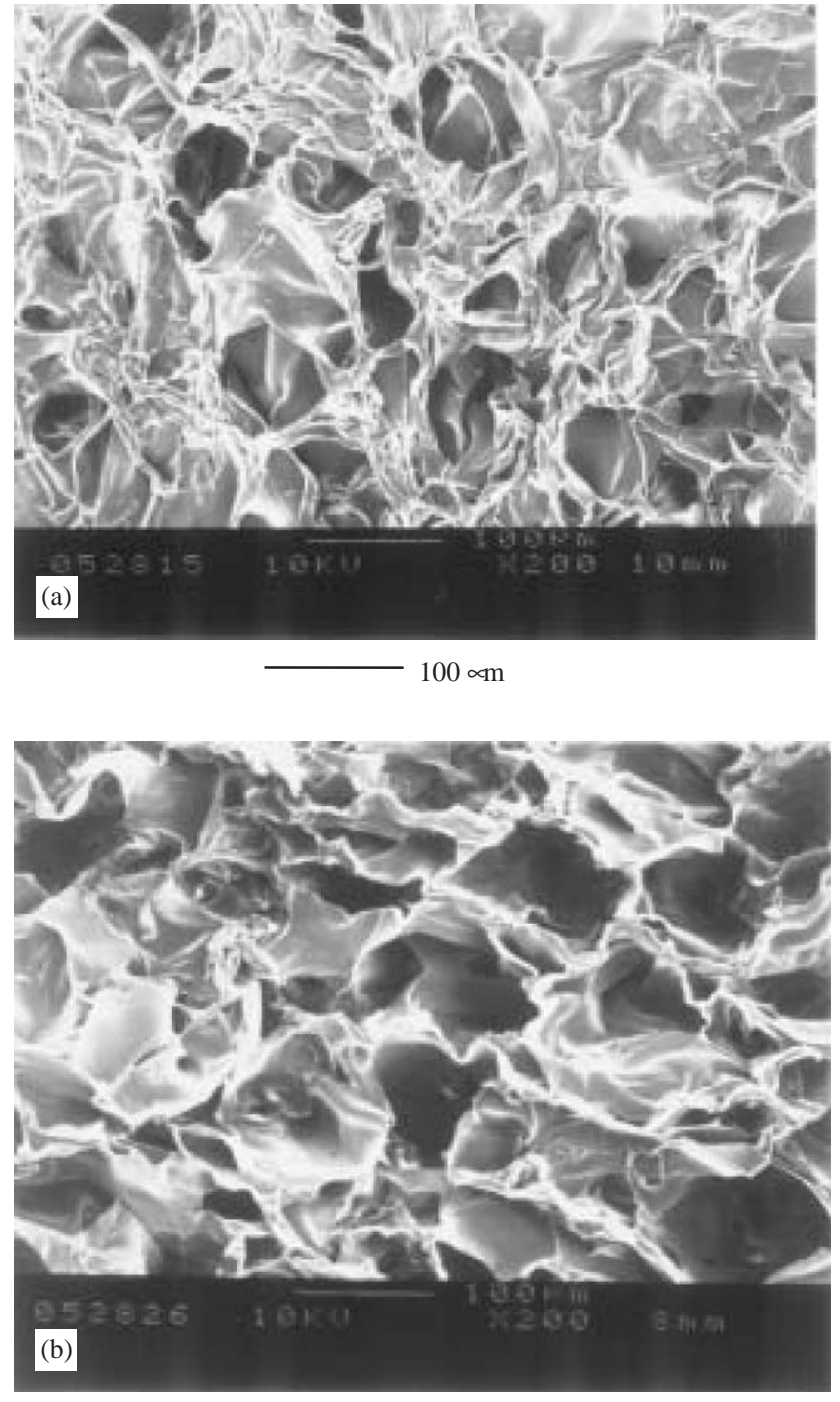

$100 \mu \mathrm{m}$

Fig. 4. Morphology of the chitosan scaffold prepared by the freezegelation method (SEM). (a) Surface and (b) cross-section.

structure during the removal of solvent. It should be noted that, when a commercial freeze-dryer was used, the obtained scaffolds possessed surface skin. The results indicate that the temperature in the commercial freeze-dryer was not low enough to make the polymer matrix in the demixed solution rigid enough to resist the interfacial tension caused by the evaporation of solvent. To avoid the formation of surface skin, a freeze-drying apparatus (Fig. 1) was set up in which liquid nitrogen can be added constantly to keep the drying temperature low. Then porous scaffolds without surface skin can be obtained and their morphologies are shown in Figs. 8-10. In comparison of Figs. 2-4 with Figs. 810 , it can be seen clearly that the scaffolds prepared with the methods proposed in this study are as porous as those with the freeze-drying method. On the basis of the above results, it can be said that the freeze-drying

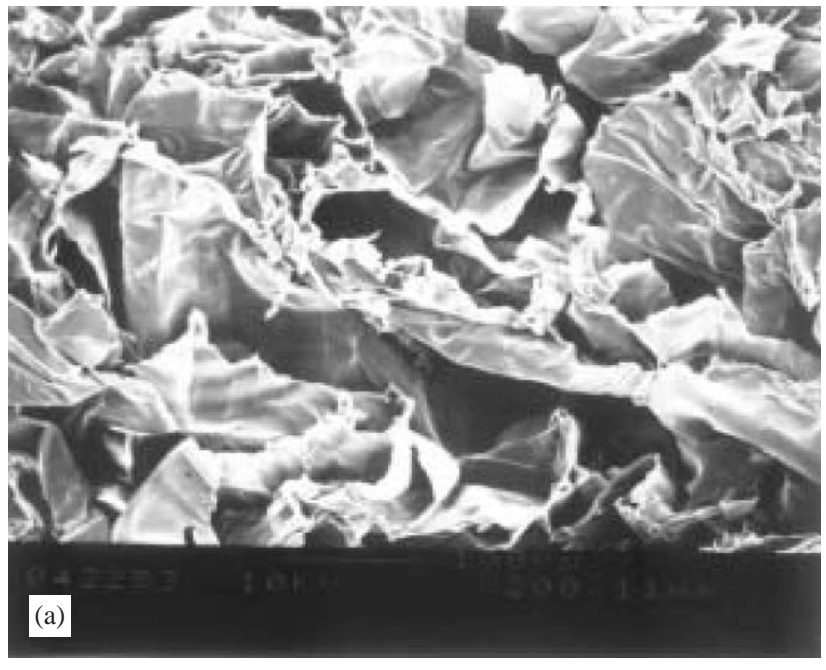

$\longrightarrow 100 \mu \mathrm{m}$

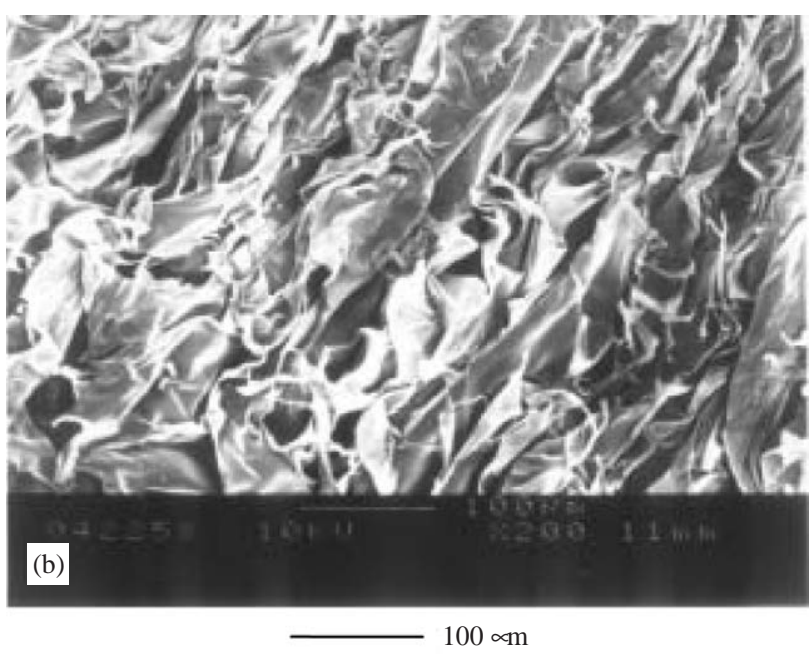

Fig. 5. Morphology of the alginate scaffold prepared by the freezegelation method (SEM) (a) Surface and (b) cross-section.

method, widely used for preparation of porous scaffolds, can be replaced with the freeze-extraction or the freeze-gelation method proposed in the present work.

\subsection{Formation of porous structure}

The roles of freeze-extraction and freeze-gelation in the formation of porous structure are discussed in this section. During the freezing stage, porous structure is generated after the phase separation of the homogeneous polymer solution. After the removal of the solvent in the demixed solution, the remained space originally occupied by the solvent, becomes pores in the scaffold. During the solvent removal, the polymer surrounding the solvent should be rigid enough to prevent pore collapse and thus to retain the porous structure. The reason of using freeze-drying to remove solvent is quite 


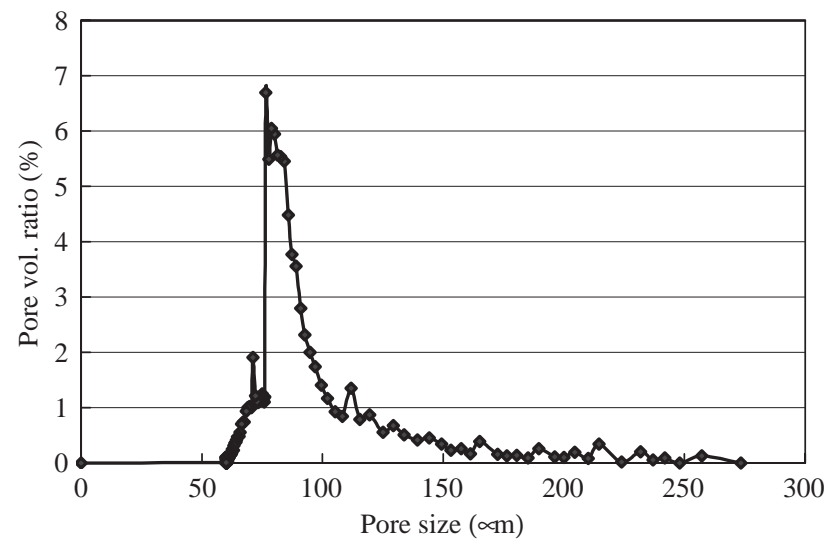

Fig. 6. Pore size distribution of PLLA scaffolds prepared by the freeze-extraction method.

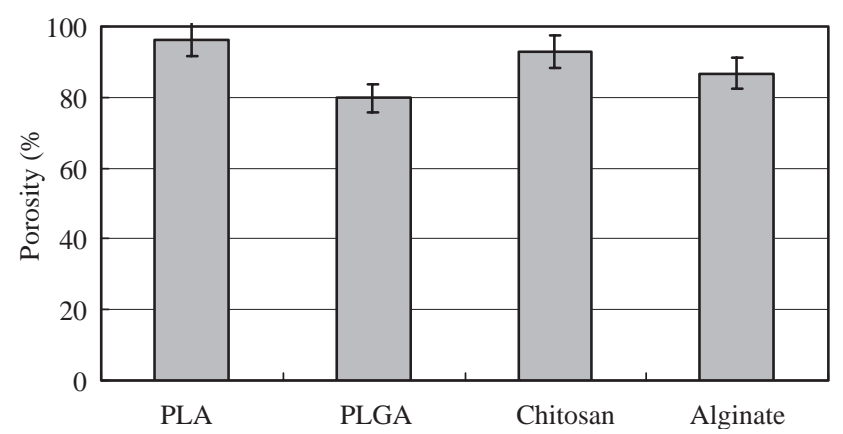

Fig. 7. Porosity of the scaffolds prepared by the freeze-extraction and freeze-gelation methods (values are the mean $\pm \mathrm{SD}, n=8$ ).

obvious: to keep the temperature low enough that the polymer-rich region would not redissolve and possesses enough mechanical strength to prevent pore collapse during drying. The results shown in Figs. 2-5 suggest that freeze-extraction and freeze-gelation can also remove solvent without destruction of porous structure, as freeze-drying does.

For the freeze-extraction method (PLLA and PLGA), the idea was to remove the solvent by extraction with a non-solvent. After the removal of solvent, the space originally occupied by solvent was taken by non-solvent and the polymer was then surrounded with the nonsolvent. Under this circumstance, even at room temperature, the polymer would not dissolve. Hence, drying at room temperature could be carried out to remove the non-solvent, leaving space that became pores in the scaffolds. It should be noted that the extraction was performed at a temperature lower than the freezing point of the polymer solution to assure that the polymer would not redissolve during the extraction process. Hence the non-solvent should have a freezing point lower than that of the polymer solution, so that it could be kept at a liquid state during extraction. The nonsolvent used in this study was ethanol aqueous solution of which the freezing point can be changed by adjusting
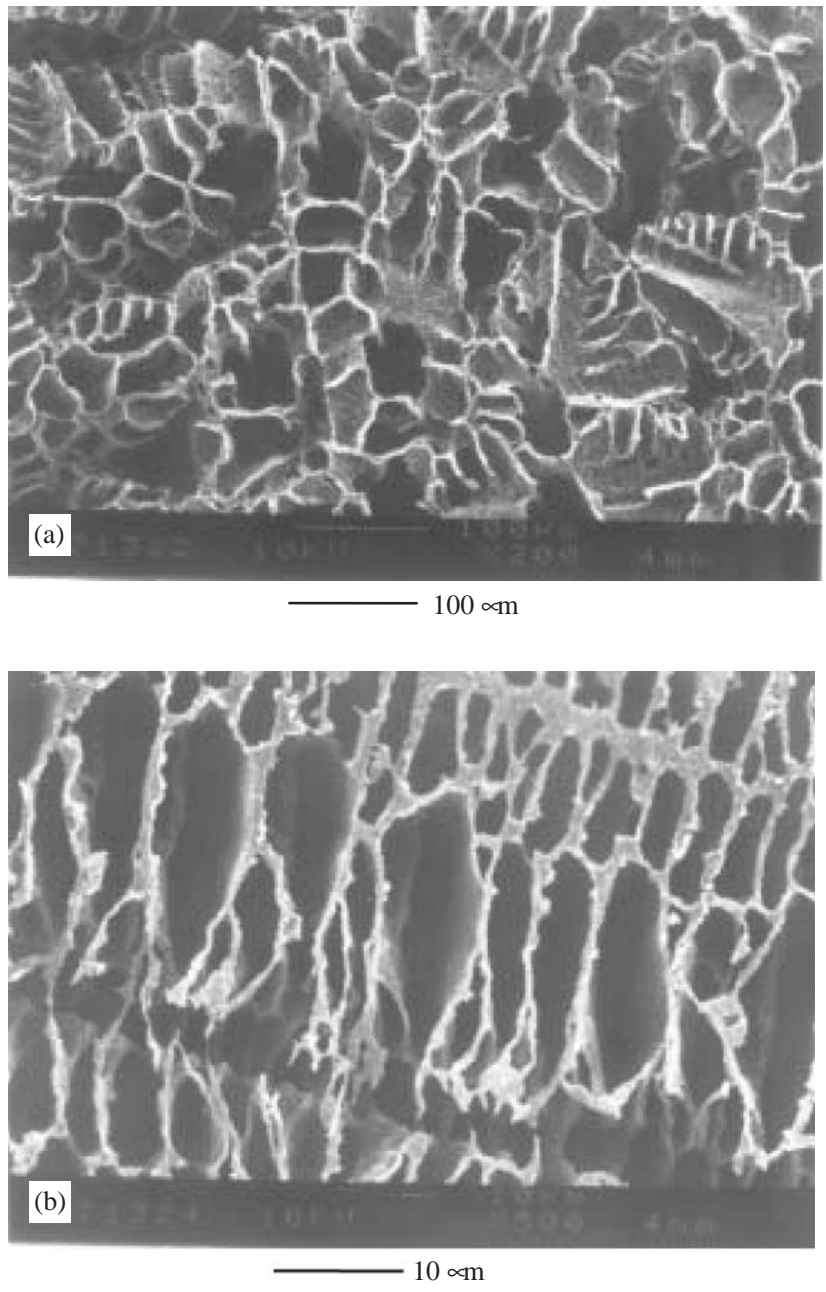

Fig. 8. Morphology of the PLLA scaffold prepared by the freezedrying method (SEM). Solvent: dioxane. (a) Surface and (b) crosssection.

its composition. With a suitable composition of ethanol aqueous solution, the non-solvent bath (ethanol-water solution) was in liquid state during the extraction of dioxane out of the frozen PLLA and PLGA solutions.

For chitosan it is not easy to extract out the solvent (aqueous solution of acetic acid) with a non-solvent. Therefore, a different method was used instead of the freeze-extraction method. The idea was not to remove the solvent but to adjust the solvent property to allow for the gelation of chitosan. It is known that chitosan would gel in an alkaline environment, on the basis of which a method was developed: immersion of the frozen chitosan solution in a solution of sodium hydroxide $(\mathrm{NaOH})$ to adjust the solution $\mathrm{pH}$ to bring about gelation. After the gelation of chitosan, the polymer did not redissolve at room temperature and was rigid enough to prevent pore collapse during drying; hence, porous scaffolds could be prepared without using freeze-drying. Similar to freezeextraction, the gelation process was carried out at a temperature lower than the freezing point of the chitosan 


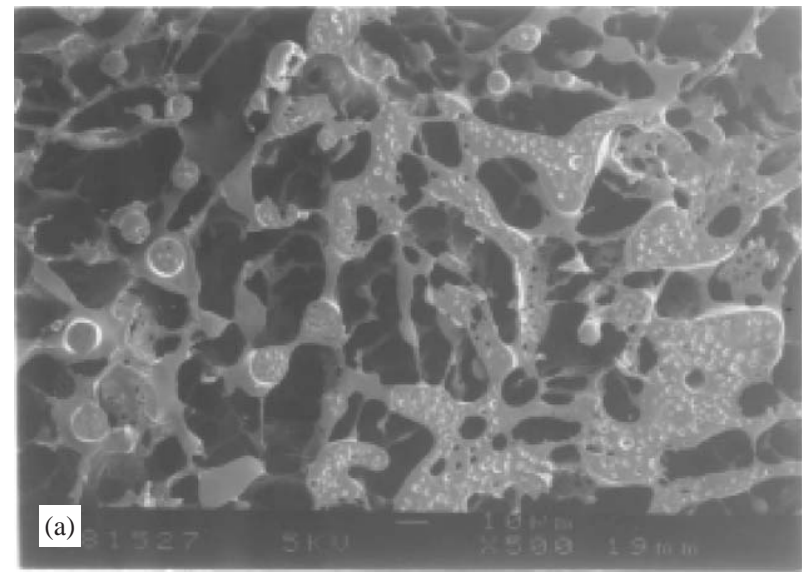

$-10 \mu \mathrm{m}$

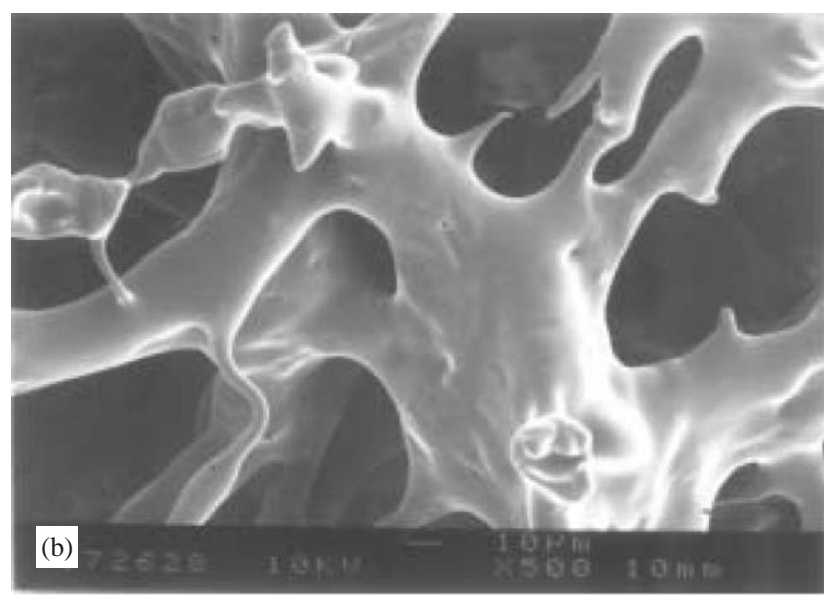

$-10 \mu \mathrm{m}$

Fig. 9. Morphology of the PLGA scaffold prepared by the freezedrying method (SEM). Solvent: dioxane. (a) Surface and (b) crosssection.

solution to prevent the redissolution of chitosan. The alkaline solution used in this study contained $\mathrm{NaOH}$, water, and ethanol. The $\mathrm{NaOH}$ aqueous solution provided the alkalinity for gelation and the addition of ethanol was to lower the freezing point.

The procedures for preparation of alginate scaffolds were similar to those for chitosan scaffolds: solution freezing, freeze-gelation, and drying at room temperature. But, the gelation method was different. The gelation of alginate was not introduced by adjustment of $\mathrm{pH}$ but by addition of calcium ion. The solution used to gel alginate consisted of calcium chloride, water, and ethanol. The aqueous solution of calcium chloride provided the calcium ion needed for alginate gelation, and ethanol was added to lower the freezing point so that freeze-gelation could be carried out.

\subsection{Advantages of freeze-extraction and freeze-gelation}

Although freeze-drying is a widely used method to prepare porous scaffolds, it is time and energy
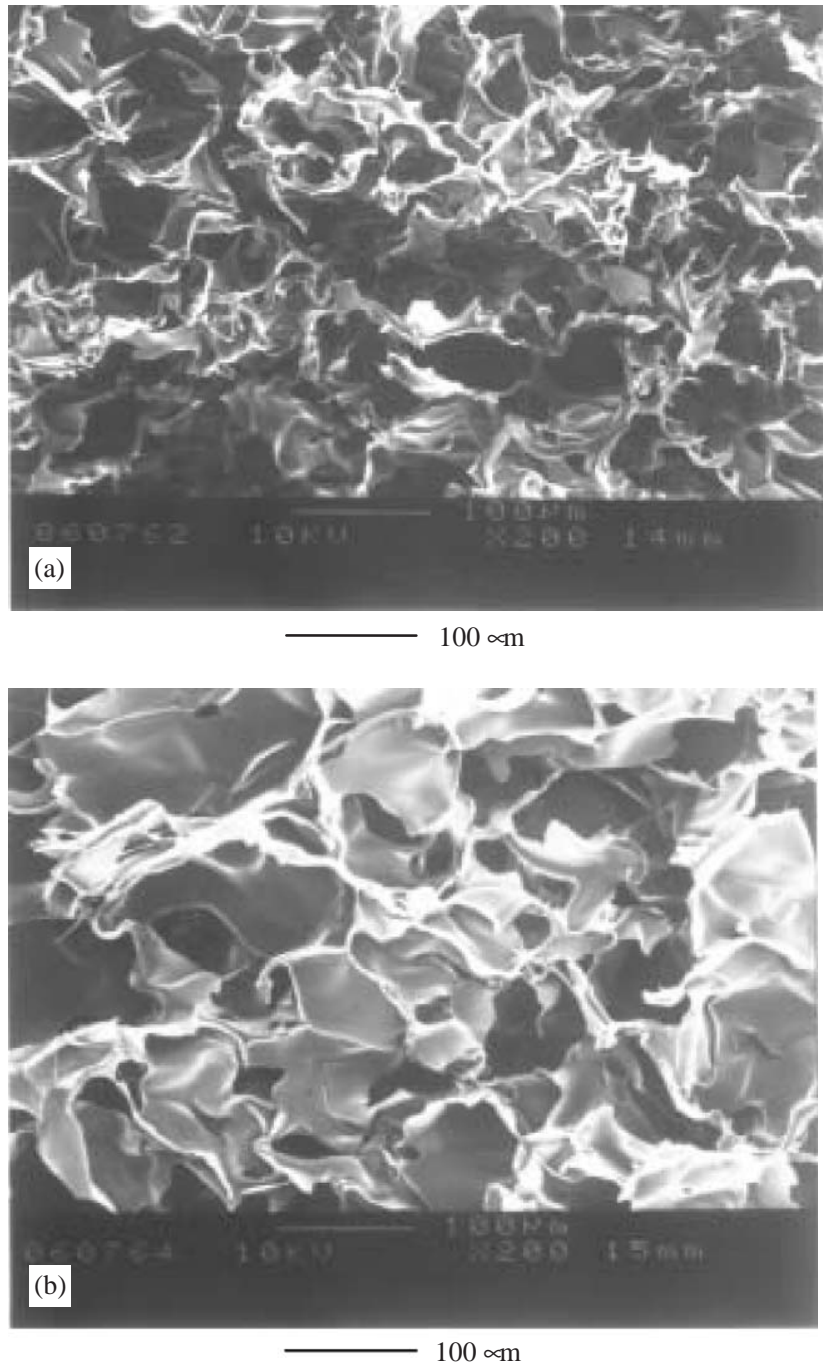

Fig. 10. Morphology of the chitosan scaffold prepared by the freezedrying method (SEM). (a) Surface and (b) cross-section.

consuming. As in our experiments, it took at least 4days to remove the solvent by freeze-drying. During this 4-day period, a lot of energy was consumed to keep vacuum and to maintain the low temperature needed for drying. By using the freeze-extraction and freezegelation methods proposed in the present work, the time and energy required for preparation of scaffolds can be greatly saved. It took only one day or less for solvent removal to prepare the scaffolds shown in Figs. $2-5$. Obviously, the proposed methods have advantages of being more efficient and economical. Another problem in using freeze-drying to prepare scaffolds is the formation of surface skin. Although it can be resolved by keeping the drying temperature low enough, the low drying temperature would make the process more time and energy consuming. By using the method presented in this work, the formation of surface skin can be avoided in a more efficient way. 
Other advantages of freeze-extraction and freezegelation are discussed in the following. The first one is related to the limitation of choosing polymer solvent. For the freeze-drying process, the solvent vapor pressure at the drying temperature (usually very low) needs to be high enough to allow its removal. Hence, the choice of solvent is quiet limited. The limitation of choosing solvent can be lifted when the scaffolds were prepared by the freeze-extraction method. As an example, DMSO cannot be used as the solvent for PLGA for the freezedrying process due to its low vapor pressure. But, for the freeze-extraction process, because DMSO can be easily extracted out by ethanol aqueous solution, it can be used as the solvent for preparation of PLGA scaffolds. The SEMs of the porous PLGA scaffolds prepared by using DMSO as the solvent are shown in Fig. 11. Another advantage of the freeze-extraction method is

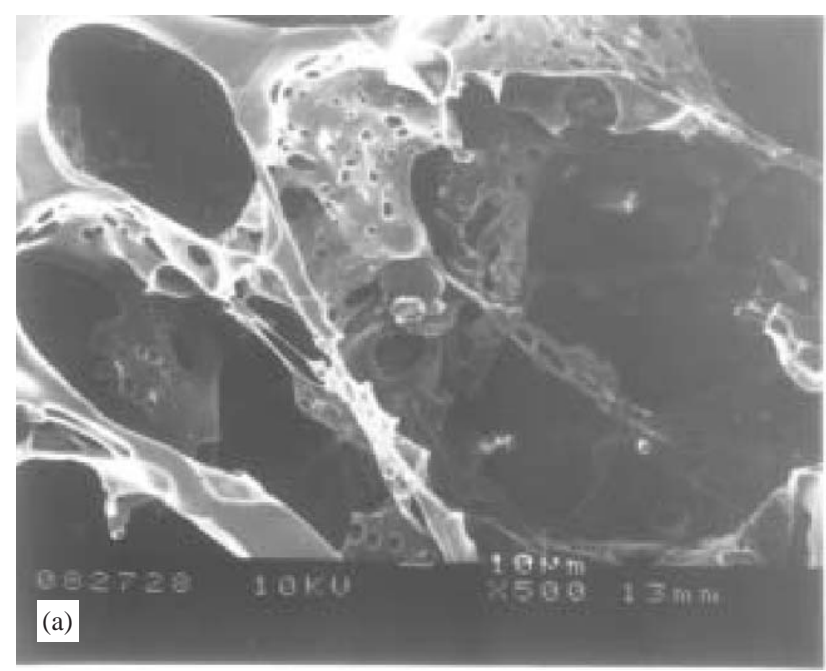

$-10 \mu \mathrm{m}$

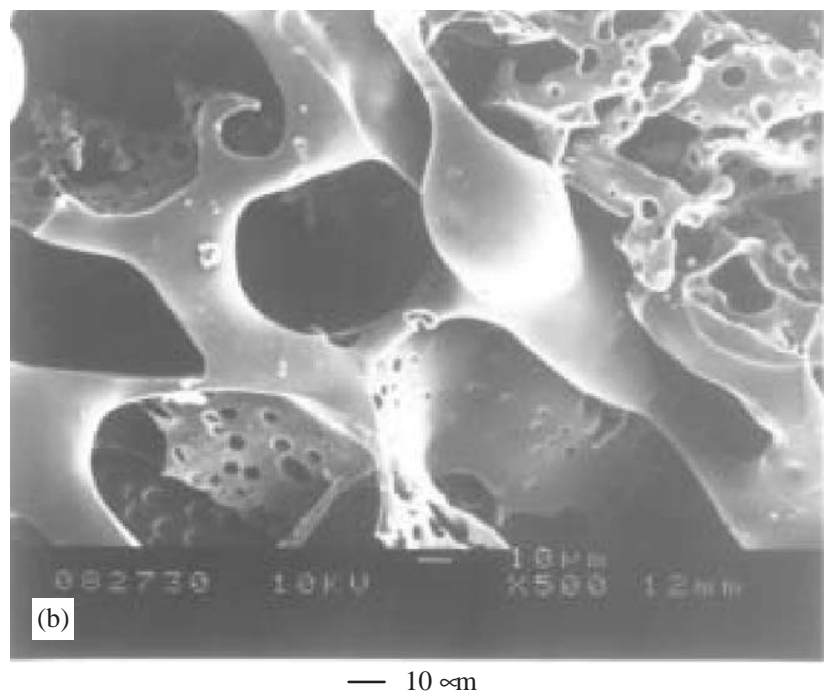

Fig. 11. Morphology of the PLGA scaffold prepared by the freezeextraction method (SEM). Solvent: DMSO. (a) Surface and (b) crosssection. about the problem of residual solvent. Due to the low efficiency of freeze-drying, the solvent might not be able to be completely removed and the residual solvent could be a problem for the application of the scaffolds. For the freeze-extraction process, the solvent is not removed by volatilization but by extraction with non-solvent. Once the amount of non-solvent is large enough, the solution can be almost completely replaced with the non-solvent. By using non-solvent which is safe for biomedical use, such as ethanol aqueous solution in this work, the problem of residual solvent can be resolved.

Moreover, compared to freeze-drying, freeze-extraction and freeze-gelation are more suitable for production of scaffolds in large scale, because they require less energy, time and space. In our experiments, scaffolds with more than $20 \mathrm{~cm}$ in diameter can be easily prepared.
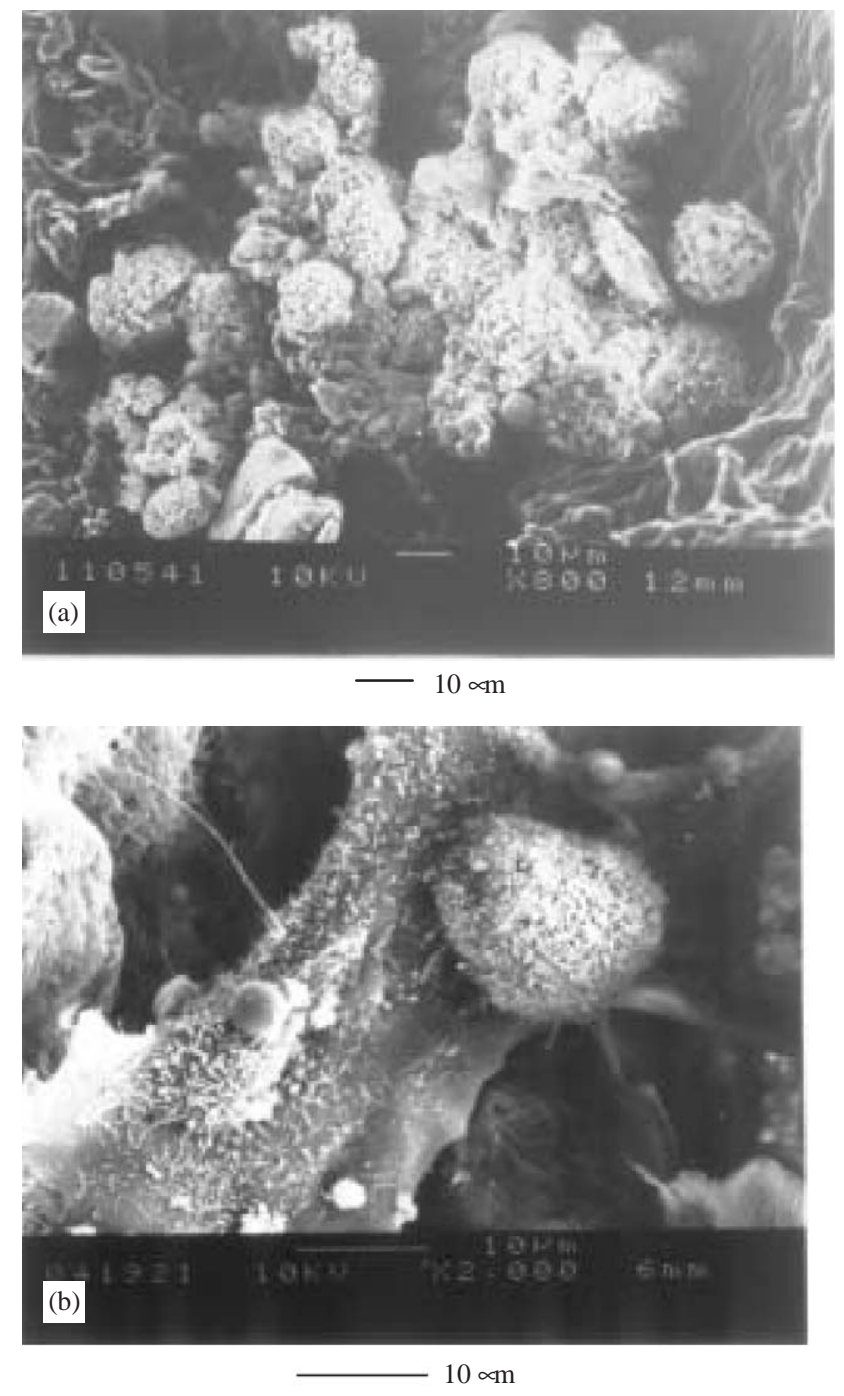

Fig. 12. Morphological characterization of ROS cells within the pores in the PLLA scaffolds prepared by the freeze-extraction method. (a) Cultured for 2 weeks $(\times 800)$ and (b) cultured for 2 weeks $(\times 2000)$ (SEM). 


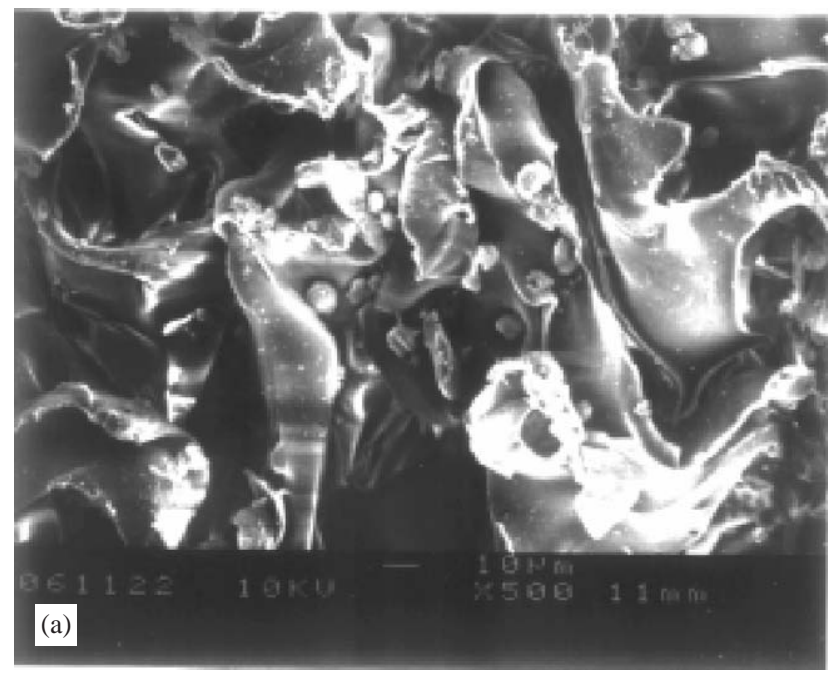

$-10 \mu \mathrm{m}$

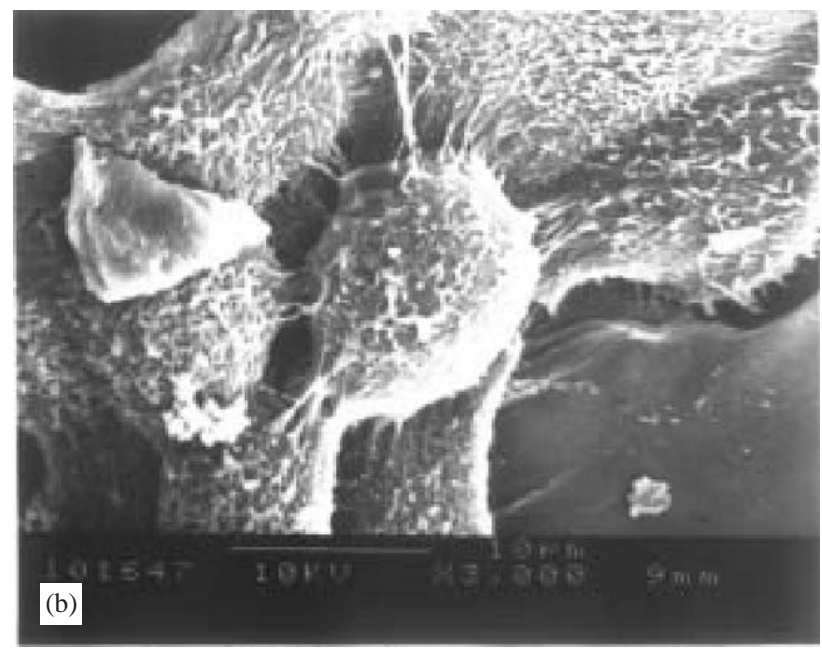

$10 \mu \mathrm{m}$

Fig. 13. Morphological characterization of ROS cells within the pores in the chitosan scaffolds prepared by the freeze-gelation method. (a) Cultured for 2 weeks $(\times 500)$ and (b) cultured for 2 weeks $(\times 3000)$ (SEM).

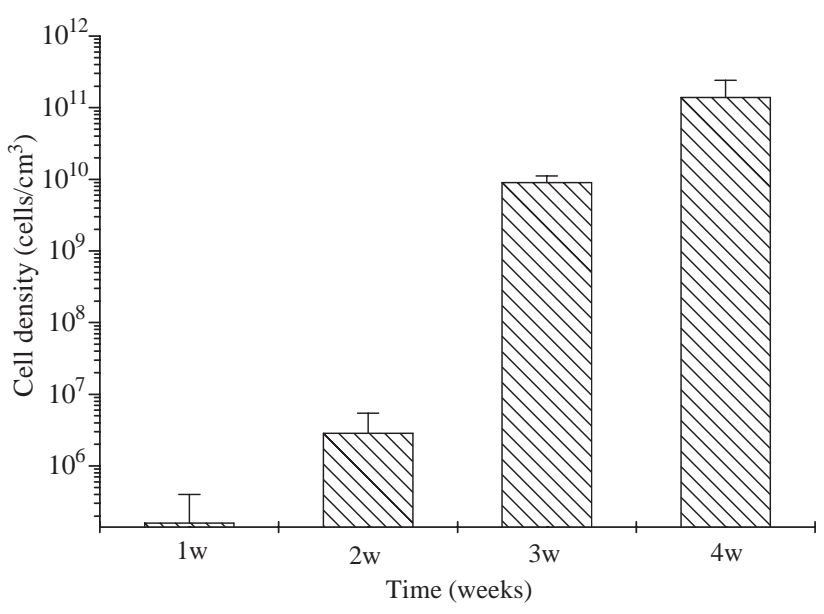

Fig. 14. ROS cell densities at different culture time intervals in the chitosan scaffolds prepared by the freeze-gelation method.

\subsection{Application of the prepared scaffolds to cell culture}

Experiments of cell culture were carried out to test the biocompatibility of scaffolds. ROS cells used in the present work are osteoblast-like cells with the ability of expressing of collagen I and osteocalcin and the ability of mineralization [19-22]. These cells are widely used to test in vitro biocompatibility of materials in vitro [2326]. The morphologies of the cultured cells within the pores of scaffolds are shown in Figs. 12 and 13. The cell density in the chitosan scaffolds at different culture time intervals were also measured and the results are shown in Fig. 14. The results indicate that the ROS cells cultured in scaffolds can attach, spread, and proliferation, demonstrating the potential applicability to tissue engineering of the scaffolds prepared by freeze-extraction and freeze-gelation.

\section{Conclusion}

It is demonstrated in the present work that porous PLLA, PLGA, chitosan and alginate scaffolds can be successfully prepared by the proposed freeze-extraction and freeze-gelation methods. The prepared scaffolds are highly porous, with porosity larger than 0.8 , and have interconnected pores ranging from 60 to $150 \mu \mathrm{m}$. Compared with the freeze-drying method, the presented methods are more time and energy efficient, with less residual solvent, and easier to be scaled up. In addition, the problem of formation of surface skin can be resolved and the limitation of using solvent with low boiling point can be lifted. The applicability to cell culture of the prepared scaffolds has also been examined. It was observed that the ROS cells cultured in scaffolds could attach, spread, and proliferate well, indicating the potential applicability to tissue engineering of the scaffolds prepared by the proposed methods.

\section{Acknowledgements}

The authors sincerely appreciate the financial support from National Science Council, Taiwan (NSC91-2216E-033-001) and the kind help on performing SEM analysis from Miss Su-Jen Ji and Professor Liang-Ping Lin, in the Advanced Instrument Center, National Taiwan University.

\section{References}

[1] Langer R, Vacanti JP. Tissue engineering. Science 1993;260: 920-6.

[2] Cao Y, Vancanti JP, Paige KT, Upton J, Vacanti CA. Transplantation of chondrocytes utilizing a polymer-cell 
construct to produce tissue-engineering cartilage in the shape of a human ear. Plast Reconstr Surg 1997;100:297-302.

[3] Freed LE, Marquis JC, Nohria A, Emmanual J, Mikos AC, Langer R. Neocartilage formation in vitro and in vivo using cells celtured on synthetic biodegradable polymers. J Biomed Mater Res 1993;27:11-23.

[4] Mooney DJ, Park S, Kaufmann PM, Sano K, Mcnamara K, Vacanti JP, Langer R. Biodegradable sponges for hepatocyte transplantation. J Biomed Mater Res 1995;29:959-66.

[5] Mikos AG, Sarakinos G, Lyman MD, Ingber DE, Vacanti JP, Langer R. Prevascularization of porous biodegradable sponges. Biotech Bioeng 1993;42:716-23.

[6] Mooney DJ, Kaufmann PM, Sano K, Mcnamara KM, Vacanti JP, Langer R. Transplantation of hepatocytes using porous biodegradable sponges. Transplant Proc 1994;26:3425-6.

[7] Schugens $\mathrm{CH}$, Grandfils $\mathrm{CH}$, Jerome R, Teyssie P, Delree P, Martin D, Malgrange B, Moonen G. Preparation of a macroporous biodegradable polylactide implant for neuronal transplantation. J Biomed Mater Res 1995;29:1349-62.

[8] Chen G, Ushida T, Tateishi T. A biodegradable hybrid sponge nested with collagen microsponges. J Biomed Mater Res 2000;51: 273-9.

[9] Chen G, Ushida T, Tateishi T. Preparation of poly (L-latide) and poly (D,L-lactic-co-glycolic acid) by use of ice microparticulates. Biomaterials 2001;22:2563-7.

[10] Ma J, Wang H, He B, Chen JT. A preliminary in vitro study on the fabrication and tissue engineering aoolications of a novel chitosan bilayer materials as a scaffold of human neofetal fibroblast. Biomaterials 2001;22:331-6.

[11] Mooney DJ, Baldwin DF, Suh NP, Vacanti LP, Langer R. Novel approach to fabricate porous sponges of poly (D,L-lactic-coglycolic acid) without the use of organic solvents. Biomaterials 1996; 17:1417-22.

[12] Harris LD, Baldwin DF, Mooney DJ. Open pore biodegradable matrices formed with gas foaming. J Biomed Mater Res 1998;42:396-402.

[13] Park YJ, Nam KH, Ha SJ. Porous poly (L-latide) membranes for guided tissue regenerarion and controlled drug delivery: membrane fabrication and characterization. J Control Release 1997; 43:151-60.

[14] Nam YS, Park TG. Porous biodegradable polymeric scaffolds prepared by thermally induced phase separation. J Biomed Mater Res 1999;47:8-17.
[15] Whang K, Thomas CH, Healy KE. A novel method to fabricate biodegradable scaffolds. Polymer 1995;36:837-42.

[16] Han MJ. Biodegradable membranes for the controlled release of progesterone. 1. Characterization of membrane morphologies coagulated from PLGA/Progesterone/DMF solutions. J Appl Polym Sci 2000;75:60-7.

[17] Schugens C, Maquet V, Grandfils C, Jerome R, Teyssie P. Biodegradable and macroporous polylactide implants for cell transplantation: I. Preparation of macroporous polylactide supports by solid-liquid phase separation. Polymer 1996;37: 1027-38.

[18] Schugens C, Maquet V, Grandfils C, Jerome R, Teyssie P. Biodegradable and macroporous polylactide implants for cell transplantation: II. Preparation of polylactide foams by liquidliquid phase separation. J Biomed Mater Res 1996;30:449-61.

[19] Majeska RJ, Rodan SB, Rodan GA. Parathyoid hormoneresponsive clonal cell lines from rat osteosarcoma. Endocrinology 1980;107(5):1494-503.

[20] Shteyer A, Gazit D, Passi-Even L, Majeska R, Gronwicz G, Rodan GA. Formation of calcifying matrix by osteosarcoma cells in diffusion chambers in vivo. Calcif Tissue Int 1986;39(1):49-54.

[21] Thiede MA, Yoon K, Golub EE, Noda M, Rodan GA. Structure and expression of rat osteosarcoma (ROS-17/2.8) alkaline phosphatase: product of a single copy gene. Natl Acad Sci 1985;85(2):319-23.

[22] Ronda GA, Majeska RJ. Phenotypic maturation of osteoblastic soteosarcoma cells in culture. Prog Clin Biol Res 1982; 110(Part B):249-59.

[23] Hou LT, Liu CM, Lei JY, Wong MY, Chen JK. Biological effects of cementum and bone extracts on human periodontal fibroblast. J Periodontol 2000;71:1100-8.

[24] Filmon R, Grizon F, Baslé MF, Chappard D. Effects of negatively charged groups (carboxymethyl) on the calcification of poly (2-hydroxyethyl methacrylate). Biomaterials 2002;23: 3053-9.

[25] Rucci N, Migliaccio S, Zani BM, Taranta A, Teti A. Characterization of the osteoblast-like cell phenotype under microgravity conditions in the NASA-approved rotating wall vessel bioreactor (RWV). J Cellular Biochem 2002;85:167-79.

[26] Akhouayri O, Proust MHL, Raeener A, Laroche N, Augusseau AC, Alexandre C, Vico L. Effects of static or dynamic mechanical stresses on osteoblast phenotype expression in three-dimensional contractile collagen gels. J Cellular Biochem 1999;76:217-30. 